\title{
Origin of the Oscillator Strength of the Triplet State of a Trion in a Magnetic Field
}

\author{
D. Sanvitto, ${ }^{1,2}$ D. M. Whittaker, ${ }^{2,3}$ A. J. Shields, ${ }^{2}$ M. Y. Simmons, ${ }^{1, *}$ D. A. Ritchie, ${ }^{1}$ and M. Pepper ${ }^{1,2}$ \\ ${ }^{1}$ Cavendish Laboratory, University of Cambridge, Madingley Road, Cambridge CB3 OHE, United Kingdom \\ ${ }^{2}$ Toshiba Research Europe Limited, Cambridge Research Laboratory, 260 Cambridge Science Park, Milton Road, \\ Cambridge CB4 OWE, United Kingdom \\ ${ }^{3}$ Department of Physics and Astronomy, University of Sheffield, Sheffield S3 7RH, United Kingdom
}

(Received 18 December 2001; published 25 November 2002)

\begin{abstract}
The dynamics of the spin-triplet trion state, under high magnetic field in a GaAs/AlGaAs quantum well, are studied using time resolved spectroscopy. The oscillator strength of the triplet transition is shown to rise with increasing electron density, in good agreement with a theoretical model where the trion interacts with excess electrons in the quantum well. This analysis suggests that the spin-triplet trion state, which is expected to be an optically "dark" state, is experimentally observable due to the interactions with the excess electrons, demonstrating that $X^{-}$cannot be regarded as an isolated three particle complex.
\end{abstract}

DOI: 10.1103/PhysRevLett.89.246805

PACS numbers: 73.21.-b, 78.47.+p, 78.55.Cr

The negative charged exciton or trion $\left(X^{-}\right)$is the semiconductor analog of the hydrogen ion $\mathrm{H}^{-}$. It is a bound state between two conduction band electrons and one valence band hole. Since its unambiguous observation in 1993 [1] $X^{-}$has been shown to dominate the optical spectra of semiconductor quantum wells (QWs) when excitons $(X)$ are generated in the presence of an excess of conduction band electrons at low temperatures. Because of the indistinguishability of the two electrons, the $X^{-}$ electronic spin configuration gives rise to singlet $\left(X_{s}^{-}\right)$and triplet $\left(X_{t}^{-}\right)$states. In the absence of an applied magnetic field the only bound state is a spin singlet, which has a binding energy of $\sim 1 \mathrm{meV}$. However, in a magnetic field of several tesla, perpendicular to the $\mathrm{QW}$, a triplet state is observable a few tenths of meV above $X_{s}^{-}[2,3]$.

Despite its status as the simplest possible many-body system, and a large amount of theoretical study, $X^{-}$is still not fully understood. In particular, the experimental observation of the $X_{t}^{-}$transition is surprising. Palacios et al. [4] showed theoretically that, in the lowest Landau level approximation, optical transitions involving the triplet state of the trion should be forbidden; i.e., $X_{t}^{-}$is a dark state. This result was generalized by Dzyubenko and Sivachenko [5], who proved that, due to a magnetic translation symmetry, the lowest energy $X_{t}^{-}$state should be dark in any quasi-two-dimensional system, provided in-plane translational invariance is maintained. Nevertheless, $X_{t}^{-}$has been observed experimentally by a number of authors, in photoluminescence (PL) [2,3,6-8], absorption [9], and reflectivity [10] spectra.

A potential solution to this puzzle was proposed by Wójs et al. [11], who suggested that the observed $X_{t}^{-}$state is not the ground state, but in fact the first excited state, for which they predicted a finite oscillator strength. Very recently Yusa et al. [12] have presented PL spectra which they interpret as showing both bright and dark triplet states, with the dark state visible only in the fractional quantum Hall (FQHE) regime at $20 \mathrm{mK}$. However, Riva et al. [13] and Peeters et al. [14] have obtained very good fits to the magnetic field dependence of the experimental singlet and triplet energies in a $300 \AA \mathrm{QW}$, using the dark $X_{t}^{-}$state. When they calculate the bright state in this wide well, they find it is unbound. More theoretical work needs to be done to determine which of these calculations is correct.

Another possible explanation for the experimental observation of the $X_{t}^{-}$, suggested in Ref. [5], is that translational invariance in the QW is broken by disorder, in the form of irregularities in the interfaces or a strong electric potential from the remote impurities. This lifts the magnetic translation symmetry, turning $X_{t}^{-}$into an optically active state. However, $X_{t}^{-}$transitions are seen with similar strength in samples which are known to contain different amounts of disorder. Furthermore $X_{t}^{-}$is also observed in samples where the trions have been shown unambiguously to be delocalized $[15,16]$. This implies that the oscillator strength cannot come mainly from the disorder.

In this Letter we present experimental results which show that the $X_{t}^{-}$oscillator strength depends on the background electron density in the QW. This suggests that the scattering of $X_{t}^{-}$with extra electrons can lift the magnetic translational symmetry in a similar way to scattering with disorder in the quantum well. We present a simple theoretical model of the interacting trionelectron system, which reproduces the measured electron density dependence of the $X_{t}^{-}$oscillator strength and supports our identification of the dark state. This contrasts with the calculations of Ref. [11], where the $e^{-}-X_{t}^{-}$ interaction is considered in the FQHE regime, which give an oscillator strength for the dark $X_{t}^{-}$at least 10 times smaller than $X_{s}^{-}$.

Our sample is a $300 \AA \mathrm{GaAs} / \mathrm{Al}_{0.33} \mathrm{Ga}_{0.67} \mathrm{As}$ QW remotely doped with $10^{17} \mathrm{~cm}^{-3} \mathrm{Si}$ donor over $200 \mathrm{~nm}$ of the 
upper barrier, spaced $60 \mathrm{~nm}$ away from the QW in order to minimize the roughness in the Coulomb potential due to the doping layer. The sample is gated with a semitransparent NiCr Schottky gate which is biased with respect to the QW Ohmic contact, allowing the electron density within the well to be varied from few $10^{9}$ to $\sim 10^{11} \mathrm{~cm}^{-2}$. In previous works $[15,17]$ we have shown that, by varying the electric bias between the surface gate and the GaAs QW, we can observe in the PL spectra emission from $X, X^{-}$and eventually a two-dimensional electron gas. Notice that in the absence of a back gate bias only a negligible electric field is present across the well [18]. The sample is excited nonresonantly using a modelocked Ti:sapphire laser with 1 ps pulse width and $82 \mathrm{MHz}$ repetition rate. The luminescence, dispersed in energy, is collected in $\sigma^{-}$polarization by a CCD for time integrated PL or a streak camera with an overall resolution of $\sim 20 \mathrm{ps}$ for time resolved PL. The sample is kept at a temperature of $2 \mathrm{~K}$, in a magnetic field of $8 \mathrm{~T}$ perpendicular to the QW.

Figure 1 shows the effects of the variation of excess electrons on the $X^{-}$formation in a magnetic field of $8 \mathrm{~T}$. The exciton dominates the PL spectrum at low electron concentration $\left(\sim 3 \times 10^{10} \mathrm{~cm}^{-2}\right)$, but an increase of the excess electron density enhances the trion formation rate, reducing the $X$ PL intensity and strengthening the $X_{t}^{-}$and $X_{s}^{-}$PL. $X_{t}^{-}$strengthens faster than $X_{s}^{-}$and eventually merges with $X_{s}^{-}$into the many-body emission feature. The sum of the integrated intensities of $X, X_{t}^{-}$, and $X_{s}^{-}$ is almost constant when the electron density is varied, during the $X \rightarrow X^{-}$transition, which indicates that the decay is predominately radiative and other nonradiative paths can be neglected.

The decay times of $X, X_{t}^{-}$, and $X_{s}^{-}$states have been studied as a function of electron concentration and are plotted in Fig. 2, within the range of electron densities for which we observe the transition of Fig. 1. To understand

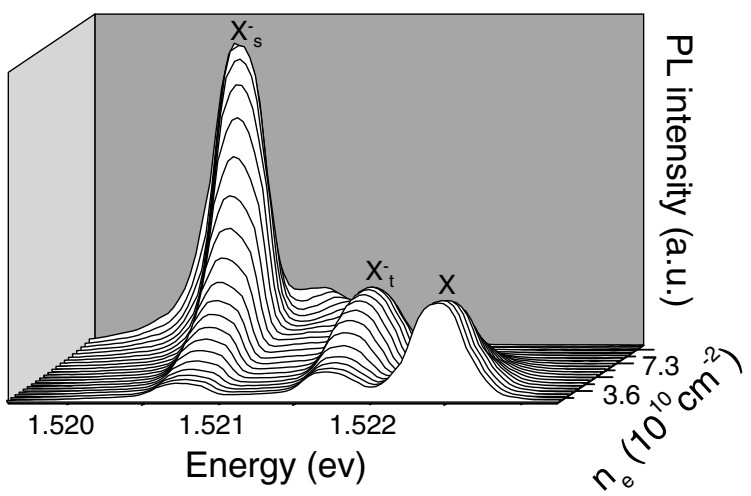

FIG. 1. Transition from $X$ to $X_{t}^{-}$and $X_{s}^{-}$in time integrated $\mathrm{PL}$ at $8 \mathrm{~T}$. Increasing the electron concentration the scattering rate from $X \rightarrow X^{-}$strengthens, resulting in a higher $X_{t}^{-}$and $X_{s}^{-}$population and stronger PL. The time integrated PL is almost constant all over the $X \rightarrow X^{-}$transition, implying negligible nonradiative decay paths. the $X, X_{t}^{-}$, and $X_{s}^{-}$decay dynamics of Fig. 2 we use a model which is shown schematically in Fig. 3. In a previous publication [17], we have demonstrated that $X^{-}$is mainly formed via excitons rather than directly from electron hole pairs. Thus the processes we include to describe the dynamics are the radiative decay of each of the states, characterized by lifetimes $\tau_{X}, \tau_{X_{t}^{-}}$, and $\tau_{X_{s}^{-}}$, and the feeding from $X$ to $X_{t}^{-}$and $X_{s}^{-}$, with lifetimes $\tau_{t}$ and $\tau_{s}$, respectively. As discussed above, the constancy of the photoluminescence efficiency allows us to exclude nonradiative processes outside the three states. We also neglect the possibility of scattering from $X_{t}^{-}$to $X_{s}^{-}$, which is slow since it necessarily involves an electron spin flip [7], and because of the low temperatures, the thermally activated reverse scattering from $X_{s}^{-}$or $X_{t}^{-}$to $X[17,19]$. Experimental support for the exclusion of all these processes comes from the observation that no PL signal is present from $X$ and $X_{s}^{-}$when $X_{t}^{-}$is excited resonantly. With these approximations, the dynamics can be simplified to $X$ independently feeding $X_{s}^{-}$and $X_{t}^{-}$. This implies that the long time decay of each of the $X^{-}$PL intensities is determined by the slower of the total decay of the $X$ $\left(1 / \tau_{X}+1 / \tau_{t}+1 / \tau_{s}\right)^{-1}$ and the radiative decay of the particular $X^{-}$state.

From Fig. 2 we see that the $X$ and $X_{s}^{-}$decay times follow a similar behavior to that observed without a magnetic field [17]. At low carrier concentration $X$ shows a long decay time which is given by its radiative lifetime $\tau_{X}$ (this is supported by direct resonant measurements of

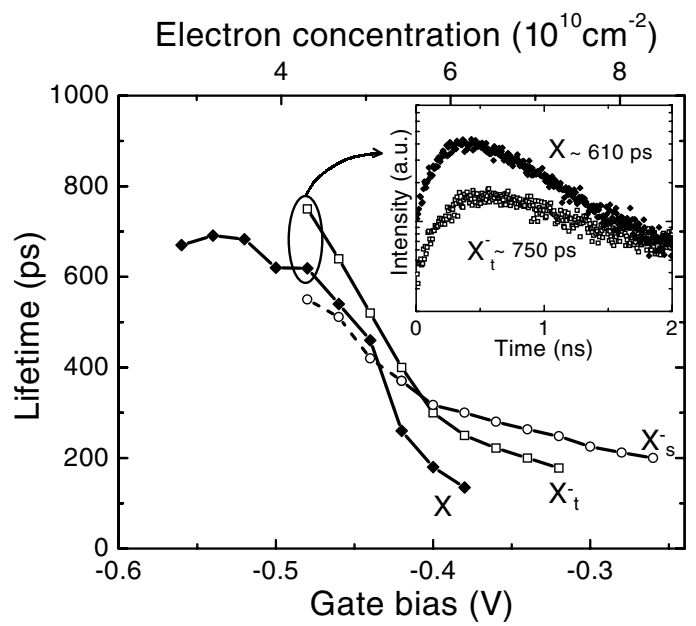

FIG. 2. Decay times as a function of gate bias (electron concentration) for $X$ (filled rhombus), $X_{t}^{-}$(open squares), and $X_{s}^{-}$(open circles). $X_{t}^{-}$is found to have a decay time longer than any other species as shown in the inset where the decay curves of $X$ and $X^{-}$are taken at an electron density of $4.3 \times$ $10^{10} \mathrm{~cm}^{-2}$. This shows that the $X_{t}^{-}$decay time corresponds to the radiative $X_{t}^{-}$lifetime. By contrast, the $X_{s}^{-}$decay time coincides with the radiative lifetime of the $X_{s}^{-}$state only at high electron densities (solid line). At lower electron concentrations the $X_{s}^{-}$decay originates from the $X$ population which feeds the $X^{-}$state (dashed line). 


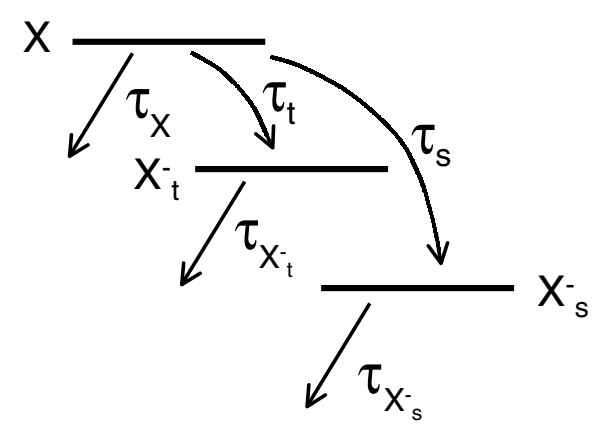

FIG. 3. Schematic diagram of the relaxation and decay dynamics of $X, X_{t}^{-}$, and $X_{s}^{-}$. See text for specific meaning of the time constants.

the $X$ lifetime $[15,20])$. With increasing electron density the $X$ decay time reduces due to a new relaxation path: the scattering of $X$ into $X^{-}$. This coincides with the appearance of $X_{s}^{-}$, at densities of $\sim 4 \times 10^{10} \mathrm{~cm}^{-2}$, with a similar decay time to that found for $X$, showing that the $X$ decay rate is slower than the $X_{s}^{-}$radiative decay time, $\tau_{X_{s}^{-}}$. Increasing the electron concentration the scattering $X \rightarrow X^{-}$strengthens, due to the higher number of electrons in the well, and the $X^{-}$formation rate increases. This is evident from the very short $X$ decay time at high electron densities. At electron concentrations of $\sim 6 \times$ $10^{10} \mathrm{~cm}^{-2}$ the $X_{s}^{-}$decay time diverges from that observed for $X$ and shows an almost constant value which correspond to the radiative $X_{s}^{-}$lifetime.

Although $X_{t}^{-}$shows a similar decrease in decay time, with increasing electron density, to that observed for the singlet state, the behavior for $X_{t}^{-}$in fact has important differences. $X_{t}^{-}$appears together with $X_{s}^{-}$but with a decay time, $\sim 750 \mathrm{ps}$, significantly longer than that of the $X$ and $X_{s}^{-}$states (see the inset of Fig. 2). This longer lifetime shows that, unlike for the $X_{s}^{-}$state, at low electron concentrations, the measured decay time is the true radiative lifetime of the $X_{t}^{-}$state. With increasing electron densities the measured $X_{t}^{-}$lifetime decreases as approximately $n_{e}^{-3}$, down to less than 200 ps at electron concentrations of $\sim 7 \times 10^{10} \mathrm{~cm}^{-2}$, beyond which the state becomes hard to resolve.

In order to understand this result, we have developed a simple model of the interaction between the $X_{t}^{-}$state and a third electron, in a perfect 2D system, using the lowest Landau level approximation. The approximation cannot describe the singlet state, which forms a bound state only if Landau level mixing is included [21]. However, over most of the experimental electron density range, we have shown that we do not measure the true singlet oscillator strength. The $X_{t}^{-}$wave function is expressed as a linear combination of sets of single particle states for the three electrons and the hole, labeled by their angular momenta in the lowest Landau level, $\left(m_{1}, m_{2}, m_{3}, m_{h}\right)$, and spins $\left(s_{1}, s_{2}, s_{3}, s_{h}\right)$. These basis states consist of a Slater determinant of the electron states, providing the correct symmetrization, multiplied by the hole state. To describe the
$X^{-}$interaction with a distant electron, the angular momentum and spin of one of the electrons $\left(m_{3}, s_{3}\right)$ is fixed, and the other two electrons and hole are placed in a triplet configuration with total angular momentum $M=m_{h}-$ $m_{1}-m_{2}=-1$, which is known to give the $X_{t}^{-}$ground state [21]. Since a large angular momentum state corresponds to an orbit of radius $r_{c} \sim \sqrt{2\left(m_{3}+1\right)} l_{c}$, we regard this as the $X_{t}^{-}$interacting with an electron at distance $r_{c}$. To make a finite basis, the values of $m_{1}, m_{2}$, and $m_{h}$ are restricted to the range $|m|<80$. The bare Coulomb interaction is then diagonalized within this basis, to find the ground state of the interacting $X^{-}$-electron system.

The oscillator strength, $f$, is obtained by applying the optical dipole operator, which destroys one of the electrons and the hole, and computing the overlap with all possible two-electron final states, also in the lowest Landau level [22]. We neglect the effect of the Coulomb interaction in the final state, so these final states are simple symmetrized pairs of single particle levels. Since we do not know how the $X$ to $X_{t}^{-}$scattering process selects the different Zeeman sublevels, we average over these. However, it should be noted that the behavior of each of the possible spin configurations is qualitatively similar.

In Fig. 4, the calculated oscillator strengths for the $X_{t}^{-}$ state are plotted as a function of electron concentration $\left(n_{e}=r_{c}^{-2}\right)$. The simulation shows that the $X_{t}^{-}$oscillator strength increases as approximately the cube of the electron density within the well, in good agreement with our experimental observation of the decay rate.

Figure 4 also shows the experimental decay rates, plotted as symbols with the right-hand scale. A quantitative comparison between theory and experiment is difficult to perform, because the measured decay rate is that of

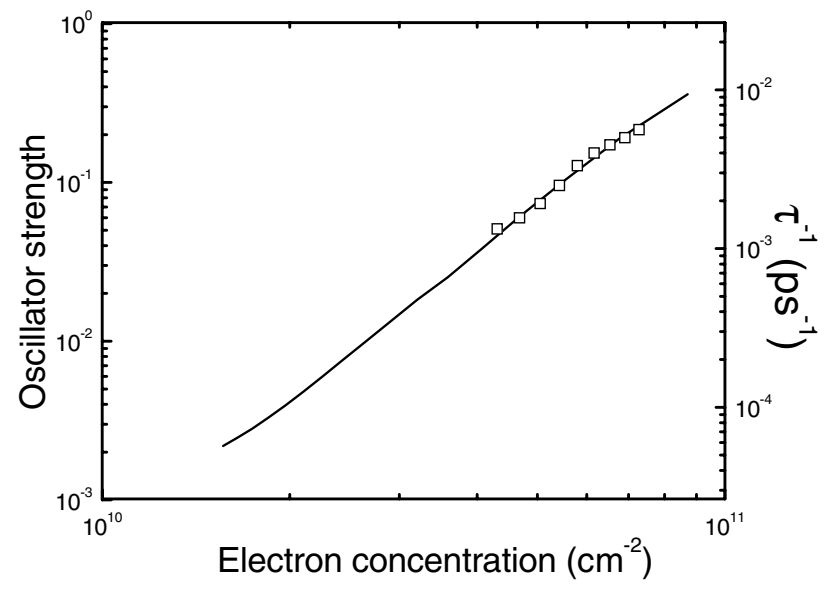

FIG. 4. The calculated oscillator strength $(f)$ of $X_{t}^{-}+e^{-}$, plotted against the surrounding electron concentration (electron distance from $X_{t}^{-}$). The model includes the electronelectron exchange interaction. The calculated lines are compared with the experimental $X_{t}^{-}$decay rates (symbols, right-hand scale). 
the $X_{t}^{-}$population, which requires a knowledge of the feeding and decay rates of all the degenerate set of $X_{t}^{-}$ states. Although such an analysis has been performed without a magnetic field [15,23], we are not aware of a similar treatment with a field present. However, we expect the low temperature dependence to be of the form $\tau_{X_{t}^{-}}^{-1}=$ $\Gamma_{0} f$, where $\Gamma_{0}$ is a constant decay rate. Fitting our experimental data gives $\Gamma_{0}=0.027 \mathrm{ps}^{-1}$, which is similar to the value of $0.016 \mathrm{ps}^{-1}$ we obtain using the formalism of Ref. [15] at zero field.

The results in Fig. 4 show that the spin-triplet state of the trion is effectively a dark state in the absence of interaction with the surrounding electrons, i.e., in our device at low carrier concentration (the calculated oscillator strength falls to values $\lesssim 10^{-3}$ at electron densities below $10^{10} \mathrm{~cm}^{-2}$ ). Then, for increasing population in the well, the $X_{t}^{-}$feels the presence of the other electrons and as a result becomes optically active. It is for this reason that it is possible to observe emission from the trion triplet state, which otherwise would be optically forbidden.

Our agreement between the experimental triplet lifetime and the numerical values calculated for the oscillator strength is in contrast with what obtained in Ref. [11]. This is because the latter model considers the LaughlinHalperin correlations, in the FQHE regime, between the $X_{t}^{-}$state and the surrounding electrons, which suppress the scattering processes giving oscillator strength to the dark state. Such correlations are not expected for the temperatures $(2 \mathrm{~K})$ used in the present experiment, too high for the FQHE, particularly in our wide QW (300 $⿱$ ) $)$ where the stability of the correlated state is doubtful even at zero temperature. This justifies our use of an uncorrelated wave function, along with its successful prediction of the experimental behavior. We also note that Ref. [11] predicts that the bright $X_{t}^{-}$oscillator strength decreases with electron density, showing that this is not the state we observe experimentally.

In conclusion, these results show that $X^{-}$cannot be regarded as an isolated three-body complex. In particular, we show that, in an applied magnetic field, the spin-triplet $X^{-}$state lifetime displays a strong dependence on electron concentration $\sim n_{e}^{-3}$. This is in good agreement with a model we develop for the $X_{t}^{-}$-electron interaction. We suggest that this result explains why the lowest energy $X^{-}$ spin triplet is experimentally observed despite the theoretical prediction that it should be a forbidden transition. We put this forward as an alternative explanation to recent suggestions that the observed triplet state is actually a higher energy bright state, coexisting with the dark state. We hope our work will stimulate more detailed calculations of excess carrier effects upon both the singlet and the triplet charged exciton oscillator strength.

We thank Nigel Cooper for helpful comments about the calculations described in this Letter.
*Permanent address: School of Physics, University of New South Wales, Sydney 2052, Australia.

[1] K. Kheng, R. T. Cox, Merle Y. d'Aubigné, F. Bassani, K. Saminadayar, and S. Tatarenko, Phys. Rev. Lett. 71, 1752 (1993).

[2] A. J. Shields, M. Pepper, M.Y. Simmons, and D. A. Ritchie, Phys. Rev. B 52, 7841 (1995).

[3] G. Finkelstein, H. Shtrikman, and I. Bar-Joseph, Phys. Rev. B 53, 1709 (1996).

[4] J. J. Palacios, D. Yoshioka, and A. H. MacDonald, Phys. Rev. B 54, 2296 (1996).

[5] A. B. Dzyubenko and A. Yu. Sivachenko, Phys. Rev. Lett. 84, 4429 (2000).

[6] S. Glasberg, G. Finkelstein, H. Shtrikman, and I. BarJoseph, Phys. Rev. B 59, R10 425 (1999).

[7] M. Hayne, C. L. Jones, R. Bogaerts, C. Riva, A. Usher, F. M. Peeters, F. Herlach, V.V. Moshchalkov, and M. Henini, Phys. Rev. B 59, 2927 (1999).

[8] T. Vanhoucke, M. Hayne, M. Henini, and V.V. Moshchalkov, Phys. Rev. B 63, 125331 (2001).

[9] D. Gekhtman, E. Cohen, A. Ron, and L. N. Pfeiffer, Phys. Rev. B 54, 10320 (1996).

[10] G. Kioseoglou, H. D. Cheong, H. A. Nickel, A. Petrou, B. D. McCombe, and W. Schaff, Phys. Rev. B 61, 4780 (2000).

[11] A. Wójs, J. J. Quinn, and P. Hawrylak, Phys. Rev. B 62, $4630(2000)$

[12] G. Yusa, H. Shtrikman, and I. Bar-Joseph, Phys. Rev. Lett. 87, 216402 (2001).

[13] C. Riva, F. M. Peeters, and K. Varga, Phys. Rev. B 63, 115302 (2001).

[14] F. M. Peeters, C. Riva, and K. Varga, Physica (Amsterdam) 300B, 139 (2001).

[15] D. Sanvitto, R. A. Hogg, A. J. Shields, D. M. Whittaker, M. Y. Simmons, D. A. Ritchie, and M. Pepper, Phys. Rev. B 62, R13 294 (2000).

[16] D. Sanvitto, F. Pulizzi, A. J. Shields, P. C. M. Christianen, S. N. Holmes, M. Y. Simmons, D. A. Ritchie, J. C. Maan, and M. Pepper, Science 294, 837 (2001).

[17] D. Sanvitto, R. A. Hogg, A. J. Shields, M. Y. Simmons, D. A. Ritchie, and M. Pepper, Phys. Status Solidi (b) 227, 297 (2001).

[18] A. J. Shields, F. M. Bolton, M. Y. Simmons, M. Pepper, and D. A. Ritchie, Phys. Rev. B 55, R1970 (1997).

[19] G. Finkelstein, V. Umansky, I. Bar-Joseph, V. Ciulin, S. Haacke, J.-D. Ganiére, and B. Deveaud, Phys. Rev. B 58, 12637 (1998).

[20] R. A. Hogg, D. Sanvitto, A. J. Shields, M. Y. Simmons, D. A. Ritchie, and M. Pepper, Physica (Amsterdam) 272B, 412 (1999).

[21] D. M. Whittaker and A. J. Shields, Phys. Rev. B 56, 15185 (1997).

[22] Similar calculations with a distinguishable third electron in a fixed state give a negligible oscillator strength for the $X_{t}^{-}$, a consequence of the hidden symmetry of Ref. [4]. In our calculation, the combination of the proper symmetrization and the fixed third electron breaks this symmetry.

[23] A. Esser, E. Runge, R. Zimmerman, and W. Langbein, Phys. Rev. B 62, 8232 (2000). 